\title{
Distribution of Neutron Resonance Widths
}

\author{
Hans A. Weidenmüller \\ Max-Planck-Institut für Kernphysik, \\ 69120 Heidelberg Germany \\ *E-mail: Hans.Weidenmueller@mpi-hd.mpg.de
}

\begin{abstract}
Recent data on neutron resonance widths indicate disagreement with the Porter-Thomas distribution (PTD). I discuss the theoretical arguments for the PTD, possible theoretical modifications, and I summarize the experimental evidence.

Keywords: random matrices; chaos; Porter-Thomas distribution; neutron widths.
\end{abstract}

\section{Why a Statistical Theory? A Reminder}

Isolated resonances observed in the scattering of very slow neutrons (with energies typically below $10 \mathrm{keV}$ ) on medium-weight and heavy nuclei have a mean spacing of about $10 \mathrm{eV}$ and an average total width of about 1 $\mathrm{eV}$. The resonances correspond to particle-instable excited states of the compound nucleus occurring at about 6 to $8 \mathrm{MeV}$ excitation energy, with several million excited states lying between the ground state and the resonances. Although the individual quantum numbers of the resonances may be known experimentally, the number of states involved is so huge that theory cannot be expected either now or in the forseeable future to be able to determine the positions, quantum numbers, and widths of these resonances. The only viable theoretical access to their properties is a statistical one where the description of individual resonance properties is replaced by statements about the distribution of resonance parameters (spacings of resonance energies, neutron widths, gamma widths). That approach goes back to E. Wigner and is based upon random-matrix theory, see the review. ${ }^{1}$

\section{Purpose}

Random-matrix theory (RMT) predicts that the reduced neutron widths $\Gamma_{n}^{0}$ (obtained from the measured partial neutron widths by removing the ap- 
propriate kinematical factor) follow the Porter-Thomas distribution (PTD) $P(y)$, a $\chi^{2}$ distribution with one degree of freedom $(\nu=1)$ given by

$$
P(y)=\frac{1}{(2 \pi y)^{1 / 2}} \exp \{-y / 2\}
$$

where $y$ is the ratio of $\Gamma_{n}^{0}$ and its average value. The PTD was successfully tested ${ }^{2}$ many years ago for the Nuclear Data ensemble. ${ }^{3}$ However, a recent test ${ }^{4}$ of the PTD in the Pt isotopes has shown that the PTD must be rejected there with 99.997 per cent significance. And a re-analysis ${ }^{5}$ of the Nuclear Data Ensemble (1245 partial neutron widths) rejects the PTD with 98.17 per cent significance. The actual analysis and the way these conclusions are arrived at are discussed in the next talk by P. E. Koehler. Here I focus on a review of the underlying theory and of the available computational and experimental evidence, with emphasis on the question: Where does the theory or the analysis possibly fail? I will not only discuss neutron widths but summarize (i) the theoretical basis for the random-matrix prediction and supporting evidence from the nuclear shell model; (ii) the special assumptions that go beyond RMT and are used to derive the PTD; (iii) the experimental evidence.

\section{Random-Matrix Theory and Evidence from the Nuclear Shell Model}

RMT is a statistical theory that yields the generic spectral properties of bound quantum systems with fixed symmetry. For a time-reversal invariant system, the Hamiltonian matrix $H$ (dimension $N \rightarrow \infty$ ) for states with fixed quantum numbers (spin and parity) is real and symmetric. The matrix elements are assumed to be random variables. Except for a normalization factor, their distribution is given by

$$
\exp \left\{-\left(N / \lambda^{2}\right) \operatorname{Trace}\left(H^{2}\right)\right\} \mathrm{d}[H] .
$$

Here $\lambda$ is a parameter that has the dimension energy and determines the mean level spacing. The symbol d $[H]$ stands for the product of the differentials of the independent matrix elements. Obviously each matrix element has a Gaussian distribution centered at zero. The distribution (2) is invariant under orthogonal transformations of Hilbert space and referred to as the Gaussian Orthogonal Ensemble (GOE). Transforming Eq. (2) to the diagonal representation of $H$ with eigenvalues $E_{\mu}, \mu=1, \ldots, N$ one finds

$$
\exp \left\{-\left(N / \lambda^{2}\right) \sum_{\mu} E_{\mu}^{2}\right\} \prod_{\mu<\nu}\left|E_{\mu}-E_{\nu}\right| \prod_{\sigma} \mathrm{d} E_{\sigma} \mathrm{d}[O] .
$$


The factor $\mathrm{d}[O]$ is the Haar measure of the orthogonal group in $N$ dimensions. The probability density (3) is the product of a term which depends only upon the eigenvalues and another that depends only upon the eigenfunctions. Therefore, eigenfunctions and eigenvalues are statistically independent. The factor $\mathrm{d}[O]$ implies that for $N \rightarrow \infty$, the projections of the eigenfunctions onto an arbitrary fixed vector in Hilbert space have a Gaussian distribution and that, therefore, the squares of these projections follow the PTD. Thus, the PTD is based upon orthogonal invariance only and is seen to be tantamount to a complete mixing of the states in Hilbert space. Correlations between eigenvalues are determined by the factors $\prod_{\mu<\nu}\left|E_{\mu}-E_{\nu}\right| \prod_{\sigma} \mathrm{d} E_{\sigma}$ and are not discussed here.

Does the RMT prediction for strong mixing of the eigenstates carry over to a realistic nuclear model like the shell model? Like RMT that model is a theory of bound states but differs from RMT in three important respects. (i) The model is governed by a mean field; (ii) the residual interaction of the model is a few-body and, usually, a two-body interaction; (iii) the complexity of angular-momentum coupling restricts applications of the model to numerical investigations. As for item (i), the single-particle states within a major shell are not degenerate. Even in the absence of any residual interaction, the many-body states of the model are, therefore, not degenerate either. For instance, in the middle of $s d$-shell, the resulting many-body spectrum extends over several $10 \mathrm{MeV}$. Strong mixing of all these states is possible only for a sufficiently strong residual interaction. As for item (ii), in RMT all states interact via independent matrix elements while a residual two-body interaction has vanishing matrix elements between all Slater determinants which differ in the occupation numbers of more that two single-particle states. As for item (iii), an analytic extrapolation of shell-model results to very large matrix dimension is not possible. One resorts to schematic models instead.

Early work on the distribution of eigenvector components in the shell model $^{6,7}$ showed deviations from the Gaussian form that could be traced to items (i) and (ii) above. There is an overabundance of small and of very large and a corresponding lack of medium-sized components reflecting incomplete mixing of the basis states of the shell model. An extensive test ${ }^{8}$ of statistical properties of eigenfunctions of the shell model focused on the information entropy $S_{k}$, a measure for the mixing of basis shell-model states of fixed spin and parity. With $W_{k \mu}$ denoting the square of the expansion coefficient of the normalized $k^{\text {th }}$ eigenfunction of the shell model (ordered by eigenenergy) in the basis $|\mu\rangle$ of shell-model states, $S_{k}$ is defined as 
$S_{k}=-\sum_{\mu} W_{k \mu} \ln W_{k \mu}$. For 12 nucleons in the $s d$-shell coupled to spin $J=2$ and isospin $T=0(N=3276$ states $)$ and interacting via a standard residual two-body interaction, $S_{k}$ approaches the GOE value only in the center of the spectrum, see Fig. 1.

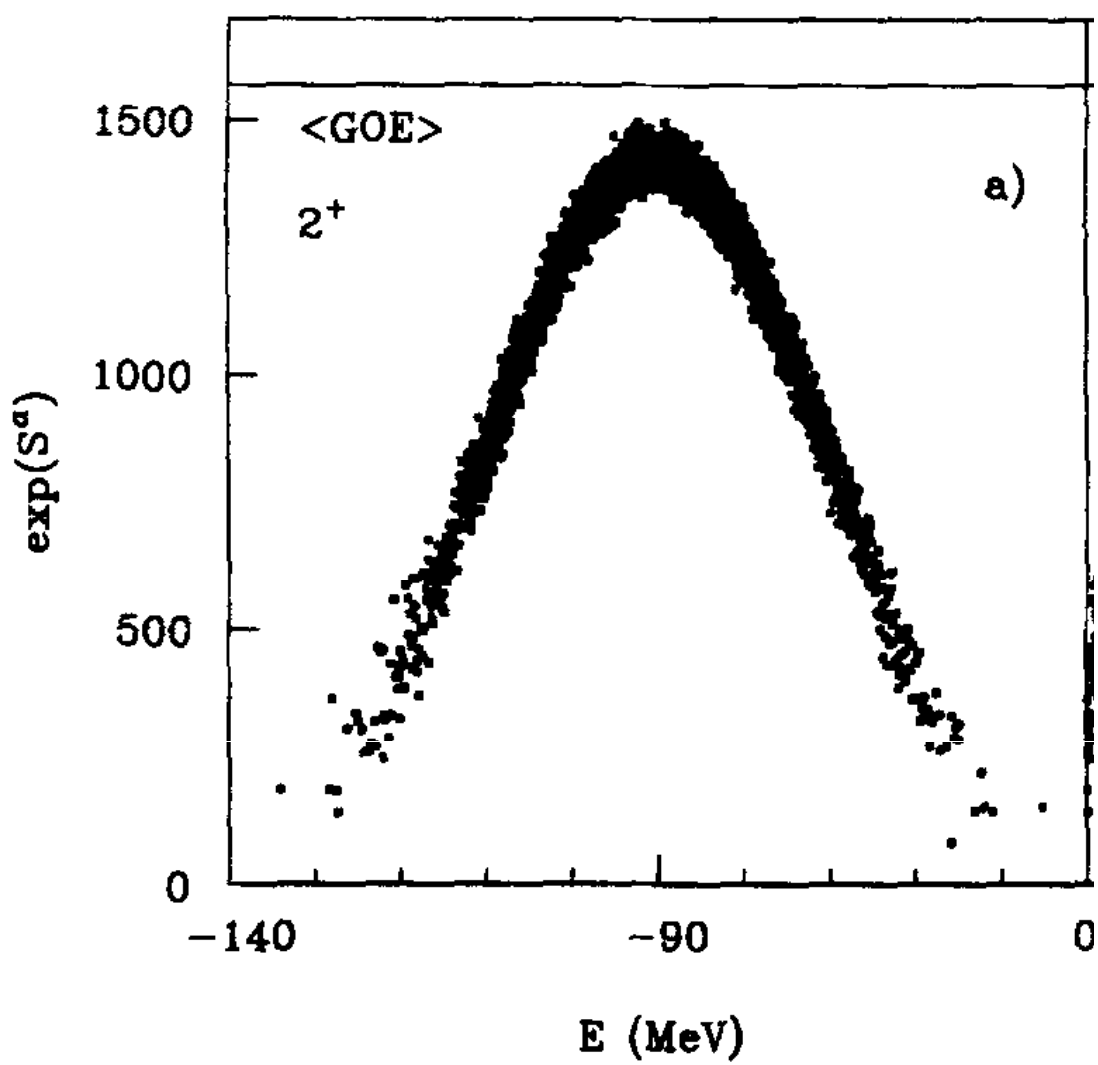

Fig. 1. Exponential of the information entropy versus eigenenergy. The solid horizontal line represents the GOE limit. Mixing of the eigenfunctions is seen to be nearly complete near the center of the shell but not in the tails. From Ref. ${ }^{8}$

It is difficult to extrapolate these results to the large matrix dimension $\left(N \approx 10^{6}\right)$ appropriate for resonances at neutron threshold. French ${ }^{9}$ used the central-limit theorem to argue that eigenvector components have a Gaussian distribution. Semi-analytical work ${ }^{10}$ suggests that strong mix- 
ing of eigenfunctions is a generic property of the shell model. For totally degenerate single-particle states, a generic two-body interaction leads to complete mixing in the large $N$ limit. ${ }^{11}$ The critical strength at which a two-body interaction produces chaos in a system with non-degenerate single-particle states was estimated ${ }^{12}$ in a schematic model and shown to be given by the average distance between directly coupled many-body states. For the $s d$-shell nuclei typical non-diagonal elements of the residual interaction are of the order of several $100 \mathrm{keV}$, i.e, are comparable to the spacing of single-particle states.

In summary, the evidence from the shell model is somewhat mixed but points to complete mixing of eigenfunctions in the large $N$ limit. That is also supported by the fact that in dynamical systems with few degrees of freedom, RMT is tantamount to quantum chaos, and quantum chaos (as opposed to integrability) presents the generic case. That should also apply to nuclei.

\section{Widths}

RMT is a theory for bound states. Additional assumptions are invoked when the coupling to the $s$-wave neutron channel is considered. The eigenstates $|k\rangle$ of RMT become resonances with widths

$$
\Gamma_{k} \propto|\langle\chi(E)|V| k\rangle|^{2} .
$$

Here $\chi(E)$ is the antisymmetrized product of the wave function of the target nucleus and a neutron $s$-wave scattering state at energy $E$, and $V$ is the nuclear interaction. At face value, $\Gamma_{k}$ is the square of the projection of the state $|k\rangle$ onto some fixed vector and should, therefore, follow the PTD. However, that argument has the following limitations. (i) The expression (4) is perturbative and holds only for strongly isolated resonances (average total width $\ll$ average resonance spacing $d$ ). (ii) Resonances occur at different neutron energies $E$. The argument breaks down if the neutron scattering wave function changes significantly with $E$ over an interval of order $d$. The kinematic $s$-wave penetration factor $\sqrt{E}$ is a trivial example. (iii) Within any dynamical model, the wave function of the target nucleus with $A-1$ nucleons is an eigenfunction of the same many-body Hamiltonian that has eigenstates $|k\rangle$ in the $A$-body system. Specifically, for a shell-model with a random two-body interaction, both $|\chi\rangle$ and $|k\rangle$ change with the interaction and are, in a statistical sense, therefore correlated. That fact violates the assumption that $|k\rangle$ is projected onto a fixed vector. The correlation comes on top of possible deviations from the GOE due to the distribution of 
components of shell-model eigenvectors as discussed in Section 3. All three points have been treated in the literature.

(i) With increasing strength of the coupling to the channel(s), the PTD is deformed. When an RMT Hamiltonian is coupled to $M$ channels, ${ }^{13}$ deviations from the PTD are already seen for $M=1$ and strong coupling. These get even bigger for $M=2$, see Fig. 2. When the superradiant state is removed from the data, the distribution of the remaining components for the single-channel case is quite close to the PTD except for intermittent values of the coupling strength. ${ }^{14}$ For the actual data on neutron widths analyzed in Refs. ${ }^{4,5}$ the effect seems marginal.

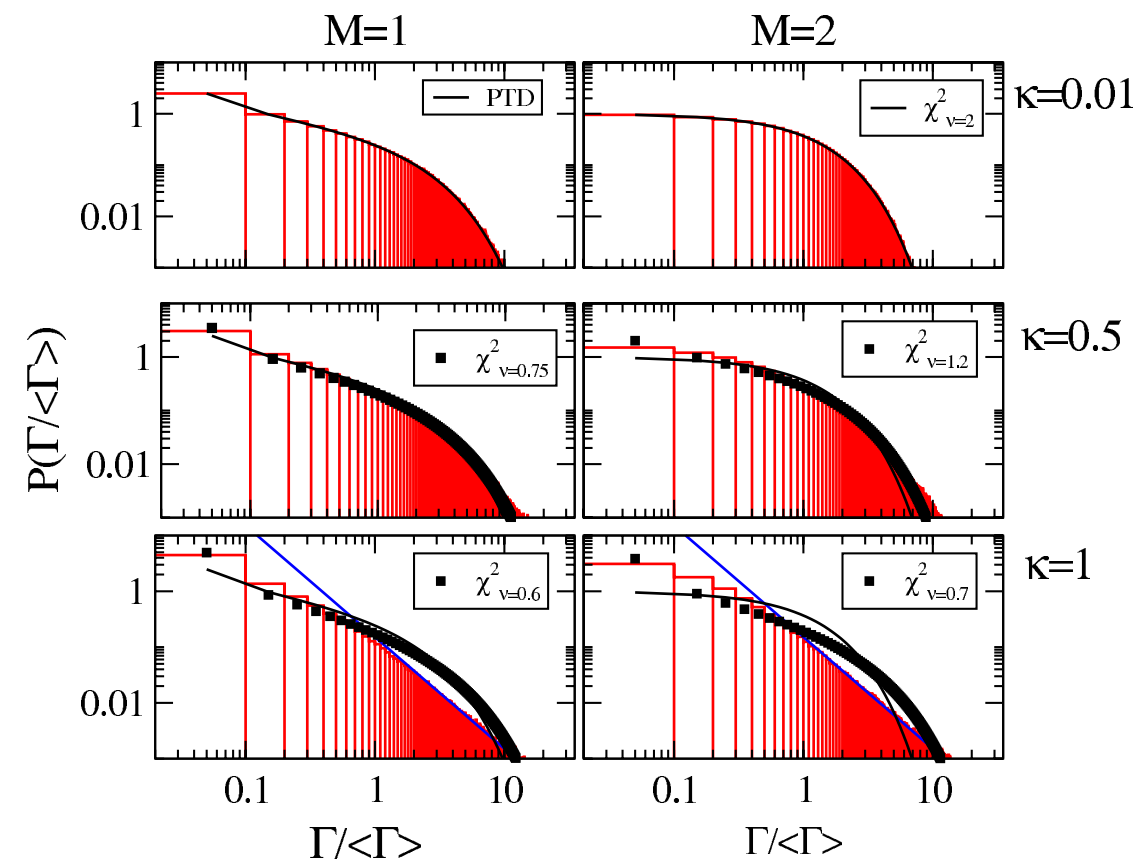

Fig. 2. (Color online) Width distributions for $M=1$ and $M=2$ channels and for various strengths $\kappa$ of the channel coupling without removal of the superradiant state. The solid lines give the PTD. From Ref. ${ }^{13}$

(ii) A secular dependence of $\Gamma_{k}$ on resonance energy beyond the $s^{-}$ wave penetration factor $\sqrt{E}$ may be due to a single-particle resonance near threshold. ${ }^{15}$ As mass number $A$ increases, single-particle $s$-wave states are pulled into the shell-model potential. When one of these is close to threshold 
it modifies the energy dependence of $\Gamma_{k}$, and when it is within $5 \mathrm{keV}$ from threshold the entire $R$-matrix analysis of the data is called into question. The Pt isotopes investigated in Ref. ${ }^{4}$ are close to a maximum of the $s$-wave strength function. That maximum corresponds to the $4 s$ single-particle state becoming bound. However, the resulting correction factor does not remove the discrepancy with the PTD distribution for the Pt isotopes ${ }^{16}$ and it is considered unlikely that the $4 s$ single-particle state is within 5 $\mathrm{keV}$ from threshold. ${ }^{16}$ For the Nuclear Data Ensemble (where most nuclei are far from maxima of the $s$-wave strength function) the correction factor is irrelevant. A doorway state above but close to neutron threshold would likewise cause a secular variation of the $\Gamma_{k}$ and would distort the PTD. That possibility seems not to have been studied yet. In any case, a secular energy dependence of $\Gamma_{k}$ beyond the $\sqrt{E}$ factor is not expected to occur uniformly for all mass numbers.

(iii) The influence of a correlation between the wave function of the target nucleus and the resonance eigenstates was studied ${ }^{14}$ in a schematic model with 7 particles $(N=11440)$ with a random two-body interaction and for several strengths of the coupling to the channel. The amplitude distribution in Fig. 3 (after removal of the superradiant state) shows deviations from the Gaussian form for all coupling strengths. For zero coupling (dashed line) there is no correlation and the effect is the same as discussed for the shell model in Section 3. As the coupling is turned on, the fraction of big components is increased at the expense of the small ones. The differences with the PTD persist. It is not clear whether these results persist or are washed out as the matrix dimension is increased from $10^{4}$ to $10^{6}$ or $10^{7}$ as would be appropriate for neutron resonances.

\section{Empirical Evidence}

Three types of tests of the distribution of eigenvectors have been performed.

(i) Statistical independence of eigenvalues and eigenvectors. This prediction of RMT is not affected by assumptions on the coupling of the eigenvectors to the channels. A test of that prediction is, therefore, stronger than is comparison with the PTD. For the Nuclear Data Ensemble, the value of the correlation coefficient was found to be consistent with zero. ${ }^{2}$ A more general later test ${ }^{17}$ supports statistical independence. Both tests confirm RMT.

(ii) Gaussian distribution of decay amplitudes. The variable in the PTD is $y_{k}=\Gamma_{k}^{0} /\left\langle\Gamma_{k}^{0}\right\rangle$. The average value $\left\langle\Gamma_{k}^{0}\right\rangle$ may be affected by a few large values that are statistically irrelevant. A measure that is less sensitive to 


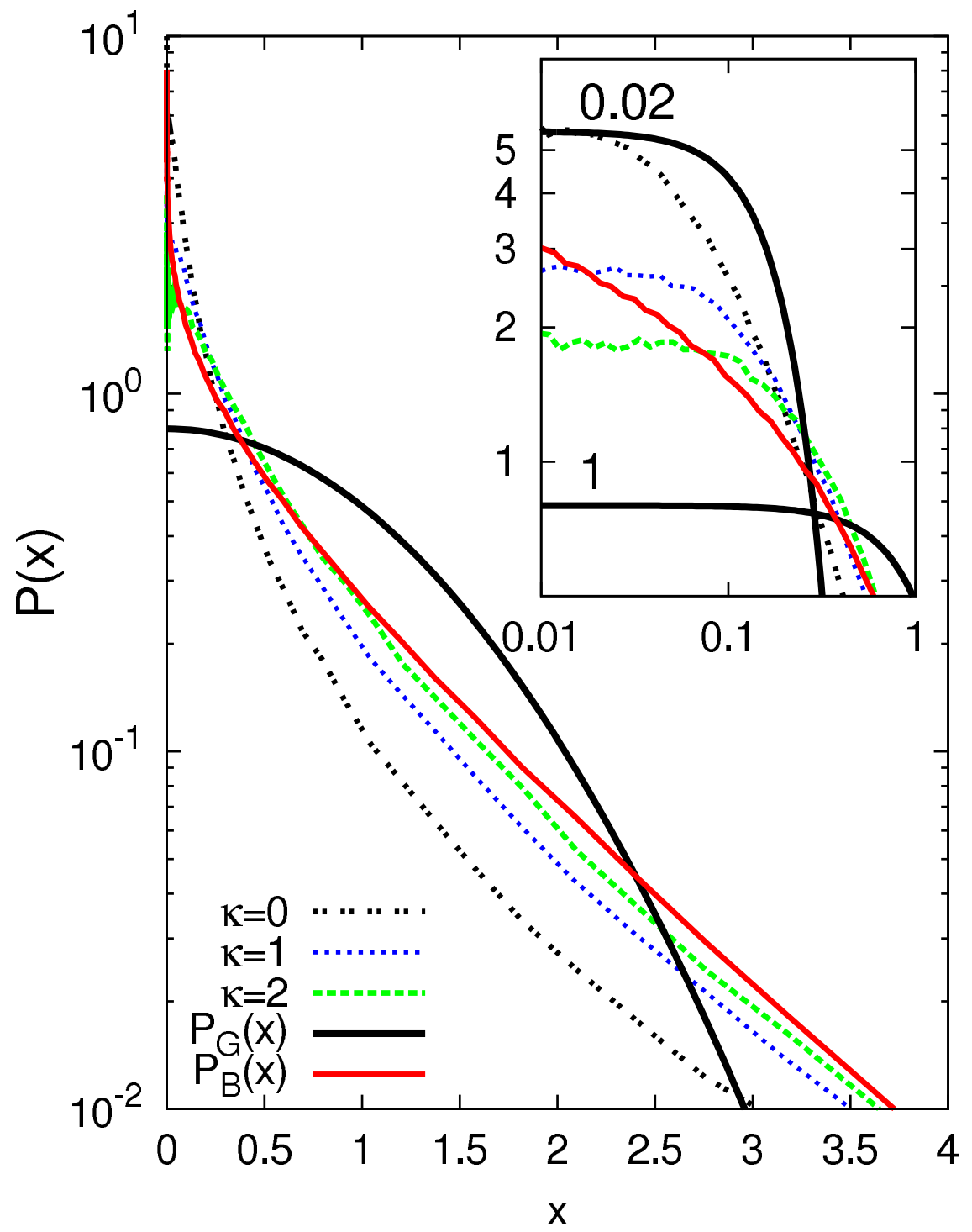

Fig. 3. (Color online) Normalized distribution of decay amplitudes versus magnitude for different coupling strengths $\kappa$. The solid black line is the Gaussian. From Ref. ${ }^{14}$

$\left\langle\Gamma_{k}^{0}\right\rangle$ compares the correlation coefficient of amplitudes (including relative phases) for decay into two different channels with the correlation coefficient of the corresponding intensities. ${ }^{18}$ For a Gaussian distribution, the second 
coefficient is equal to the square of the first. That test is not available for neutron decay widths (where only the intensities are known) but has been applied ${ }^{19,20}$ to inelastic proton resonance data. Measurements of the (p, p' $\gamma$ ) angular correlation yielded 1117 reduced width amplitudes for nuclei in the mass 50 region. Within statistics, the square of the amplitude correlation coefficient was found to be equal to the intensity correlation coefficient. This is considered a very precise test of the Gaussian distribution of amplitudes and, thus, of the PTD for widths.

(iii) Neutron widths. There are several problems in the data analysis. In a given nucleus, the number of neutron resonances is typically small, so that data from several nuclei must be combined. Resonances with small widths may remain undetected. Small fluctuations of the cross section may be mistakenly identified as resonances. Weak $s$-wave resonances are indistinguishable from strong $p$-wave resonances. These difficulties are addressed in the next talk by P. Koehler.

The early analysis ${ }^{2}$ of the Nuclear Data Ensemble showed very good agreement with the PTD, including the search for the best $\chi^{2}$ distribution. Exceptions were found ${ }^{21}$ in ${ }^{232} \mathrm{Th}$. To eliminate the above-mentioned uncertainties, a modified analysis ${ }^{22}$ used only reduced widths larger than a prescribed value. A maximum-likelihood approach was used to determine the parameter $\nu$ in the distribution $y^{(\nu / 2)-1} \exp \{-y / 2\}$ with $y$ as defined above. Here $\nu=1$ corresponds to the PTD. For 9 nuclei with masses $A>150$ and more than 45 resonances in each, the error-weighted average value of $\nu$ over these nuclei is ${ }^{22}\langle\nu\rangle=0.98 \pm 0.10$, in agreement with the PTD. The same method but with an energy-dependent cutoff and $\left\langle\Gamma_{k}^{0}\right\rangle$ as additional fit parameter was applied to the 450 resonances in the Pt isotopes and gave $\nu$ values around 0.6 so that the PTD was rejected at a significance level of 99.997 per cent. ${ }^{4}$ For nuclei in the Nuclear Data Ensemble, the same analysis gave $\nu=1.217 \pm 0.092$ so that the PTD is rejected here with a significance of 98.17 per cent. ${ }^{5}$ It is surprising that the deviations from $\nu=1$ have opposite signs in the Pt isotopes and in the Nuclear Data Ensemble, indicating a lack (preponderance) of large widths, respectively.

\section{Summary and Conclusions}

RMT, general arguments involving the central-limit theorem, entropy arguments within the shell model, and the large $N$ limit of similar other models all imply or suggest for matrices of sufficiently large dimension the validity of the PTD for the distribution of squares of eigenvector components.

Among possible causes for deviations, strong coupling to channel(s) is 
probably not significant for the actual data. A secular energy dependence of the reduced widths due to threshold effects or to a doorway state is possibly significant in some (but not all) nuclei. Insufficient mixing of shellmodel states combined with correlations between target and compoundnucleus eigenfunctions causes deviationd from the PTD. It is not clear, however, whether these effects would persist for matrices of realistically large dimension.

Among the various tests, the test for independence of eigenvalues and eigenvectors supports RMT. The test for the Gaussian distribution of proton decay amplitudes strongly supports the PTD. In contrast to earlier work, the distribution of neutron widths from the Pt isotopes strongly contradicts the PTD and so does the recent re-analysis of the distribution of neutron widths from the Nuclear Data Ensemble.

The situation is confusing because we have pieces of contradictory evidence. New data will be helpful and most welcome. But equally important is an analysis of all existing data that would employ identical criteria and use all statistical measures available. Such an analysis should be devoted to the statistical properties of both, resonance energies and resonance amplitudes (or widths). Since the Nuclear Data Ensemble was first investigated, new statistical measures have been introduced, many pieces of data have been added, and numerous quantum number assignments have been made. All of this should be taken into account. The situation calls for a concerted effort at establishing the statistical properties of nuclei.

\section{References}

1. H. A. Weidenmüller and G. E. Mitchell, Rev. Mod. Phys. 81, 539 (2009).

2. O. Bohigas, R. U. Haq, and A. Pandey, in: Nuclear Data for Science and Technology, K. H. Böckhoff, editor (D. Reidel, Dordrecht, 1983), p. 809.

3. R. U. Haq, A. Pandey, and O. Bohigas, Phys. Rev. Lett. 48, 1086 (1982).

4. P. E. Koehler et al., Phys. Rev. Lett. 105, 072502 (2010).

5. P. E. Koehler, arXiv 1001.0517 and Phys. Rev. C (accepted).

6. R. R. Whitehead et al., Phys. Lett. B 76, 149 (1978).

7. J. J. M. Verbaarschot and P. T. Brussard, Phys. Lett. B 87, 155 (1979).

8. V. B. Zelevinsky et al., Phys. Rep. 276, 85 (1996).

9. T. A. Brody et al., Rev. Mod. Phys. 53, 385 (1981).

10. T. Papenbrock and H. A. Weidenmüller, Rev. Mod. Phys. 79, 997 (2007).

11. T. Papenbrock et al., Phys. Rev. E 83, 031130 (2010).

12. Ph. Jacquod and D. L. Shepelyansky, Phys. Rev. Lett. 79, 1837 (1997).

13. G. L. Celardo et al., Phys. Rev. Lett. 106, 042501 (2011).

14. A. Volya, Phys. Rev. C 83, 044312 (2011).

15. H. A. Weidenmüller, Phys. Rev. Lett. 105, 232501 (2010). 
16. P. E. Koehler et al., arXiv 1101.4533 and Phys. Rev. Lett., submitted.

17. M. Lombardi, O. Bohigas, and T. H. Seligman, Phys. Lett. B 324, 263 (1994).

18. H. L. Harney, Phys. Rev. Lett. 53, 537 (1984).

19. J. F. Shriner, Jr., G. E. Mitchell, and E. G. Bilpuch, Phys. Rev. Lett. 59, 435 (1987).

20. J. F. Shriner, Jr., G. E. Mitchell, and E. G. Bilpuch, Z. Phys. A 332, 45 (1989).

21. L. Forman et al., Phys. Rev. Lett. 27, 117 (1971).

22. H. S. Camarda, Phys. Rev. C 49, 1391 (1994). 